\title{
Dokumentations- und Kodierprozesse im Spital: Herausforderungen und Massnahmen
}

\author{
S. Stark, S. Hölzer
}

Der Dokumentationsaufwand im Spital steigt stetig an. Das haben eine Vielzahl von Untersuchungen nachgewiesen. Vor allem klinisch tätige Ärzte und Pflegepersonen sind von der Zunahme der patientenbezogenen und administrativen Dokumentation am meisten betroffen. In einer repräsentativen Erhebung an deutschen Krankenhäusern hat das Deutsche Krankenhausinstitut (DKI) den Dokumentationsaufwand im ärztlichen und Pflegedienst in den Fachgebieten Innere Medizin und Chirurgie untersucht [1].

\section{Ergebnisse der Studie des DKI}

\section{Dokumentationsaufwand - Ärztlicher Dienst}

Die Dokumentationsarbeiten nehmen mit etwa $25 \%$ einen wesentlichen Anteil der ärztlichen Arbeitszeit in Anspruch [1]

Bei der Erhebung wurden sowohl patientenbezogene Dokumentationsarten (z.B. Erstellung von Entlassungs-, Op- und Verlegungsberichten) als auch administrative Dokumentationsarten (z.B. Verschlüsselung von Diagnosen/Prozeduren, Schriftverkehr mit den Kostenträgern) erfasst. Der Gesamtdokumentationsaufwand belief sich in der Inneren Medizin auf 194,9 Minuten = 3:15 Stunden je Arzt und Arbeitstag und in der Chirurgie 161,9 Minuten = 2:42 Stunden je Arzt und Arbeitstag.

\section{Dokumentationsaufwand - Pflegedienst}

Im Pflegedienst der Chirurgie und der Inneren Medizin benötigen die Arbeitskräfte etwa 20\% ihrer Arbeitszeit für die Dokumentation und Administration [2]

Hier wurden Daten von insgesamt fünf pflegefremden Tätigkeitsbereichen erfasst, wobei die Dokumentation und Administration nur einen Tätigkeitsbereich darstellte (ein weiterer Bereich war unter anderem der Patientenbegleitdienst). Der Ressourcenverbrauch war im Bereich der Dokumentation und Administration am grössten. In der Chirurgie benötigt eine Pflegekraft durchschnittlich 88,2 Minuten pro Arbeitstag für die Dokumentation und Administration, in der Inneren Medizin 96,5 Minuten pro Arbeitstag (etwa 20\% der Arbeitszeit bei einer 38,5Stunden-Woche).

\section{Ursachen des ansteigenden} Dokumentationsaufwandes

Die Untersuchungen haben unter anderem gezeigt, dass die Aufwandswerte der Dokumentation in den Fachabteilungen sowie bei den Ärzten und Pflegenden sehr inhomogen sind. Diese Inhomogenität ist vermutlich auf die unterschiedlichen Ablauf- und Arbeitsorganisationen in den einzelnen Spitälern zurückzuführen.

Beispiele

- Mangelnder Einsatz der vorhandenen technischen Ausstattung (z. B. Informationstechnologie) zwingt dazu, Daten häufig mehrfach zu dokumentieren.

- Externe Dokumentationsanforderungen, z. B. seitens der Gesetzgebung und der Kostenträger, verhindern eine Entlastung bei der Dokumentation.

- Dem ärztlichen und pflegerischen Personal hat man in den Spitälern in den vergangenen Jahren viele Verwaltungsaufgaben übertragen, die andere Berufsgruppen ebenso gut erledigen können.

Diese Erkenntnisse sind unseres Erachtens direkt auf die schweizerischen Spitäler übertragbar. Die weniger stringenten gesetzlichen Anforderungen zur Verantwortlichkeit der Medizinischen Dokumentation in der Schweiz erlauben eine bessere und effizientere Verteilung dieser Arbeiten. Dabei bleibt abzuwägen, wie wichtig die medizinisch-fachliche Kompetenz (z.B. bei Kodierung, Befundübermittlung usw.) bei der Erbringung der Dokumentationsleistungen bewertet wird und welche Anforderungen an die Datenqualität gestellt werden!

Auswege aus dem Dokumentationsdschungel Die Reduktion des Dokumentationsaufwandes ist in erster Linie eine spitalinterne Aufgabe, die individuelle Strategien und Lösungsansätze erfordert. Eine nachhaltige Verbesserung des Daten- und Dokumentationsmanagements benötigt tiefgreifende Struktur- und Prozessveränderungen.

Die Delegation von Dokumentationsarbeiten an andere Berufsgruppen stellt eine mögliche 
Massnahme dar. Optimal qualifiziertes Personal kann Defizite in den Arbeitsabläufen erkennen und sie beheben. Damit ist ein positiver Effekt bei Ärzten und Pflegenden zu erwarten. Sie können sich wieder auf den Schwerpunkt ihrer eigentlichen Tätigkeit am Krankenbett konzentrieren. Die Reduktion der Arbeitsbelastung ist ebenfalls vor dem Hintergrund neuerer Arbeitszeitgesetzgebung auf europäischer Ebene (EuGH) anzustreben. Gleiches gilt für die Schweiz, wo alle Assistenzärzte seit dem 1. Januar 2005 der Maximalarbeitszeit des Arbeitsgesetzes von 50 Wochenstunden unterstellt sind.

Weitere Verbesserungspotentiale liegen bei den Standardisierungen der Dokumentationsabläufe und der damit einhergehenden Mitarbeiterschulung, in der Ausstattung mit Hilfsmitteln und bei einer Verbesserung der Organisation in diesem Bereich [3].

\section{Was ist bei der Einführung von DRGs zusätzlich zu beachten?}

Mit der Einführung eines fallpauschalierten Abrechnungssystems werden die Anforderungen an die Dokumentation und Qualitätssicherung nochmals verschärft. Schlechte Dokumentation und Leistungserfassung kann in Zukunft direkt ertragsmindernd wirken. Zusätzliche Dokumentationsrichtlinien und Anforderungen werden notwendig, und vorhandene Standards, Regelwerke und Vorschriften sind zu überprüfen und gegebenenfalls anzupassen, um eine unnötige Dokumentationsbelastung zu vermeiden.

Im Hinblick auf die Einführung von DRGs sind die bereits erwähnten Ablauf- und Prozessoptimierungen unbedingt notwendig. Nur wenn die Ärzte und Pflegenden in Zukunft wieder mehr ärztlich bzw. pflegerisch tätig sind, kann der optimale Behandlungsablauf eines Patienten im Spital gewährleistet und die Qualität der Behandlung gesichert werden.

Unter einem Fallpauschalen-(DRG-)System müssen alle stationären Fälle in Bezug auf Diagnosen und relevante Prozeduren verschlüsselt werden. In einer retrospektiven Pilotstudie am Universitätsspital Zürich wurde erstmals der Zeitaufwand für die Kodierung der Behandlungen nach ICD-9-CM (CHOP) für stationäre Patienten aus der Inneren Medizin ermittelt [4]. Bei der Stichprobe handelte es sich um insgesamt 100 stationäre Fälle aus den verschiedenen Abteilungen des Departements für Innere Medizin. Bei diesen 100 Fällen wurden die Behandlungen retrospektiv anhand der sogenannten Roten Liste (verkürzte Liste von wichtigen Prozedurenkodes für medizinische Leistungen) kodiert und der benötigte Zeitaufwand erfasst. Bei 10 der
100 Fälle wurden die Behandlungen nicht nur mit der Roten Liste kodiert, sondern es wurde umfassend mit der vollständigen ICD-9-CM verschlüsselt und der Zeitaufwand davon bestimmt. Er betrug bei den Fällen, die mittels Roter Liste kodiert wurden, im Durchschnitt 5,5 Minuten pro Fall und bei denjenigen Fällen, wo die Kodierung mit der vollständigen ICD-9-CM-Liste erfolgte, im Durchschnitt 22 Minuten pro Fall.

\section{Behandlungskodierung erhöht bei $25 \%$ das durchschnittliche Fallgewicht}

Weiterhin hat man untersucht, welche Auswirkungen die Kodierung der Behandlung auf die DRG-Gruppierung hat. Hierzu wurden die 100 Fälle einmal nur anhand der Diagnosekodes in eine der möglichen DRG-Gruppen eingeteilt, und anschliessend wurden neben den Diagnosekodes auch die Kodes der Roten Liste als Gruppierungskriterium berücksichtigt. Es wurde festgestellt, dass in etwa einem Drittel der Fälle der Einbezug der Behandlungskodierung eine Veränderung der DRG-Gruppierung zur Folge hatte und sich das durchschnittliche Fallgewicht bei einem Viertel der Fälle erhöhte. Bei den 10 Fällen, die mit der vollständigen ICD-9-CM kodiert wurden, gab es keine zusätzliche Veränderung bei der DRG-Gruppierung.

\section{Schlechte Kodierung generiert finanzielle Nachteile}

Die Studienergebnisse zeigen, dass der Kodierung unbedingt Beachtung geschenkt werden muss, da andernfalls mit finanziellen Nachteilen zu rechnen ist. Dies kommt noch stärker zum Tragen, wenn zukünftig sogenannte differenzierte (refined) DRG-Systeme zum Einsatz kommen. Mit diesen gelingt eine verbesserte, leistungsgerechtere Vergütung über Spitäler aller Versorgungsstufen. Dabei muss jedoch der einzelne Fall genauer und medizinisch präziser (z.B. mit Nebendiagnosen) erfasst werden. Im Zusammenhang mit diesen Dokumentationsanforderungen wird der professionelle Einsatz von Kodierspezialisten an Bedeutung gewinnen.

\section{Aktueller Stand in den Spitälern}

Die Kodierqualität in den schweizerischen Spitälern ist sehr heterogen. Dies, obwohl bereits im Rahmen der VESKA-Statistik von H+ bis 1996 und $\mathrm{ab}$ diesem Zeitraum vom Bundesamt für Statistik flächendeckend Hauptdiagnosen und medizinische Prozeduren erfasst und weitergeleitet werden. Im Jahr 2001 wurden im Mittel etwa 1,3 Diagnosen pro stationären, administrativen Fall in den Schweizer Spitälern dokumentiert und an das Bundesamt für Statistik weiter- 
geleitet. Je nach Kanton, Grösse des Spitals und personeller Infrastruktur kann dieser Wert jedoch erheblich variieren. In einer Umfrage im Jahr 2002 [5] zeigte sich, dass nur in Ausnahmefällen zum Beispiel Komorbiditäten und Komplikationen eines medizinischen Falles systematisch als Nebendiagnosen kodiert wurden. In einer Erhebung des Schweizer Spitalverbandes H+ im Jahr 2005 in den Spitälern, die sich auf DRGs vorbereiten bzw. damit bereits arbeiten, lag die durchschnittliche Anzahl an Diagnosen pro Fall bei 4,25. Es zeigen sich jedoch weiterhin Defizite bei der Dokumentation und Kodierung von kostenrelevanten, sogenannten nichtoperativen medizinischen Behandlungen. In der möglichst genauen Erfassung der Morbidität bei den eigenen Patienten sind gerade diese kostenrelevanten medizinischen Prozeduren in Zukunft durch eine geeignete Leistungserfassung und systematische Kodierung zu berücksichtigen.

\section{Fazit aus der Sicht von $\mathrm{H}+$}

Dem Spitalverband H+ ist es ein besonderes Anliegen, den Dokumentationsaufwand in Umfang und Komplexität in beherrschbaren Grenzen zu halten. Dies bedingt, dass einheitliche Kodierregeln für den Einsatz zur Fallgruppierung in einem DRG-System und im Rahmen der medizinischen Bundesstatistik erarbeitet werden. Die Kodierung von medizinischen Prozeduren ist aus der Leistungserfassung abzuleiten, die im Spital ambulante und stationäre Leistungen beinhalten kann. Zur Entlastung des ärztlichen und pflegerischen Personals ist dort, wo es aus Effizienzgründen sinnvoll ist, der Einsatz von geschultem Fachpersonal (z.B. Kodierer) vorzusehen. Es besteht schon jetzt auf dem Arbeitsmarkt ein gewisser Mangel an diesem Fachpersonal. Er muss teilweise durch Personen aus dem Ausland ge- deckt werden. Die Möglichkeiten von Aus- und Weiterbildung in diesem Bereich sind zusätzlich zu nutzen. Spezifische Kurse bietet unter anderem das $\mathrm{H}+$ Bildungszentrum in Aarau an (www. kurse.hplus.ch/kurse/ Fachbereich: «für alle Berufsgruppen»).

$\mathrm{H}+$ als nationaler Verband der Spitäler setzt sich weiterhin dafür ein, dass vertrauliche $\mathrm{Pa}-$ tientendaten in möglichst geringem Umfang an Dritte weitergereicht werden. Was die obligatorische Datenerhebung durch das Bundesamt für Statistik betrifft, ist ein zusätzlicher Aufwand nicht mehr tragbar. Die Anonymisierung personenbezogener, schützenswerter medizinischer Daten ist bei jeder Form der Weitergabe an Dritte zu gewährleisten. Nur ein langfristig ausgerichtetes und tragbares Konzept kann hier die notwendige Datenqualität gewährleisten, die im Hinblick auf klinische Forschung, Epidemiologie und die Abrechnung von Spitalleistungen von allen Seiten gefordert wird.

\section{Literatur}

1 Blum K, Müller U. Dokumentationsaufwand im Ärztlichen Dienst der Krankenhäuser. www. dkgev.de/pdf/175.pdf. 2003.

2 Blum K. Entlastungspotenziale im Pflegedienst der Krankenhäuser. Das Krankenhaus 2003; 6:459-61.

3 Haack J. Medizinische Versorgung neu organisiert, Optimierung von Arbeitszeit und Arbeitsprozessen mit Hilfe von medizinischen Dokumentationsassistenten. www.mbhessen.de/docs/haack02.pdf. 2003.

4 Eschmann E, Mauron T, Blaser J. Aufwand und Ertrag bei der Codierung von Behandlungen für Fallpauschalen von stationären internistischen Patienten. Schweiz Ärztezeitung 2000;80(20): 1049-55.

5 Lenz M. 4. Deutsche Casemix Conference Frankfurt 2002 . 